\title{
SAÚDE MENTAL NA ATENÇÃO BÁSICA: POTENCIALIDADES PARA A CONSTRUÇÃO DE REDES DE ATENÇÃO À SAÚDE
}

\section{Maria Denise Schimith}

Universidade Federal de Santa Maria ma.denise2011@gmail.com

\section{Maria de Lourdes Denardin Budó}

Universidade Federal de Santa Maria

lourdesdenardin@gmail.com

Bruna Simon Sodré

Universidade Federal de Santa Maria enf.brusimon@gmail.com

\section{Franciele Savian Batistella}

Universidade Federal de Santa Maria

fransavian@gmail.com

\section{Dyan Jamilles Teixeira Brum}

Universidade Federal de Santa Maria

jamillesenf@gmail.com

Erro! Fonte de referência não encontrada.

Objetiva-se relatar a experiência, destacando aprendizados, limites e avanços da saúde mental na atenção básica, de um projeto de extensão desenvolvido em uma Unidade de Saúde da Família do interior do Rio Grande do Sul, Brasil, em ação desde 2008. A principal ferramenta são as visitas domiciliares, realizadas por docentes e discentes do curso de enfermagem da Universidade Federal de Santa Maria. Os resultados são apresentados em dois eixos temáticos: "ressocializar: um processo que necessita vínculo e rede", e "sequelas de uma

(1) (-) Esta obra está licenciada sob uma Licença Creative Commons. 
história de abandono". Destaca-se a importância de uma rede de atenção à saúde mental articulada e pautada nos princípios do Sistema Único de Saúde para que o eixo norteador da Reforma Psiquiátrica resgate a cidadania do usuário em sofrimento psíquico.

Palavras-chave: Modelo de formatação de artigo. Regras básicas. Revista eletrônica.

\title{
MENTAL HEALTH IN PRIMARY CARE: THE POTENTIALS FOR CONSTRUCTION OF HEALTH CARE NETWORKS
}

\begin{abstract}
It aim to report the experience highlighting learning, limitations and advances of the mental health in primary care of an extension project developed in a Family Health Unit of the interior of Rio Grande do Sul, Brazil, in action since 2008. The main tool is the home visits realized by teachers and nursing students of the nursing course at Universidade Federal de Santa Maria. The results are presented in two thematic axes: "resocialization: a process that needs bond and network", and "sequel of a desertion history." It is highlighted the importance of a network's care for mental health articulated and based on the principles of the Brazilian Unified Health System for that the guiding principle of psychiatric reform rescue the citizenship to the user in psychological distress.
\end{abstract}

Keywords: Mental health; Primary health care; Brazilian unified health system; Continuity of patient care. 
Saúde mental na atenção básica: potencialidades para a construção de redes de atenção à saúde

\section{INTRODUÇÃO}

O movimento da Reforma Psiquiátrica brasileira surgiu na década de 70 , como estratégia de crítica ao modelo de assistência manicomial prestada aos sujeitos em sofrimento psiquiátrico. Desde então, foi vista como uma transformação de saberes e valores culturais, políticos e sociais (BRASIL, 2005).

O ano de 1987 foi ímpar para a Reforma Psiquiátrica, pois realizou-se a I Conferência Nacional de Saúde Mental na cidade do Rio de Janeiro, e o primeiro Centro de Atenção Psicossocial (CAPS) do Brasil entrou em funcionamento em São Paulo. Ainda no final dos anos 80, o Deputado Paulo Delgado apresentou ao Congresso Nacional um Projeto de Lei, que somente foi sancionado em 2001 (Lei Federal $n^{\circ} 10.216$ de 6 de abril de 2001). Tal lei prevê o direito de que o indivíduo com transtorno mental seja tratado com humanidade e respeito, visando à sua reinserção na família, trabalho e comunidade (BRASIL, 2001).

Salienta-se que com a consolidação do Sistema Único de Saúde (SUS) foi idealizada a criação de uma rede de atenção à saúde mental em nosso país, caracterizada pela desinstitucionalização, a qual propõe que a assistência ao sujeito com sofrimento psíquico seja realizada por redes, compostas pelos CAPS, hospitais-dia e hospitais-noite, Unidade Básica de Saúde (UBS), Unidade de Saúde da Família (USF), dentre outros (BRASIL, 2007).

Nesse contexto, focaliza-se a atenção básica $(\mathrm{AB})$ como parte fundamental dessa rede de serviços substitutivos, e a Estratégia de Saúde da Família (ESF) foi escolhida por gestores das instâncias municipais, estaduais e federal como principal caminho para expansão e consolidação da $\mathrm{AB}$, a fim de buscar resolutividade e ampliar o impacto na situação de saúde da coletividade (BRASIL, 2011). Os usuários adstritos à AB/ESF deveriam ser reconhecidos como cidadãos com biografia particular e território existencial e geográfico conhecidos, configurando a saúde mental um eixo da ESF, uma vez que os pressupostos da Reforma Psiquiátrica convergem com o processo de trabalho desse modelo de atenção (CAMPOS et al., 2006).

Diante disso, emerge a alternativa da visita domiciliária (VD) como componente do acompanhamento a esses usuários, tendo como foco o contexto familiar e o convívio da rede social dos sujeitos em sofrimento mental (GOFFMAN, 2003). A VD constitui, assim, um instrumento facilitador na abordagem dos usuários e sua família, pois permite compreender a 
Saúde mental na atenção básica: potencialidades para a construção de redes de atenção à saúde

dinâmica familiar, bem como fornecer aos usuários suporte para a continuidade do tratamento (REINALDO, ROCHA; 2002). Essa proposta torna-se essencial, uma vez que ao adentrar no espaço privado do núcleo familiar amplia-se o entendimento do profissional de saúde em relação à família do usuário em acompanhamento e o seu contexto, aumentando as possibilidades de oferecer uma assistência adequada (LABATE, GALERA e AVANCI, 2004).

O presente artigo visa relatar a experiência de um projeto de extensão que tem como objetivos a prestação de cuidados domiciliares aos usuários com sofrimento psíquico e suas famílias, e possibilitar ao acadêmico de enfermagem vivenciar a realidade e praticar o cuidado centrado na busca da autonomia do sujeito e nas diretrizes do SUS.

\section{MATERIAL E MÉTODOS}

Trata-se de um relato da experiência acerca de ações realizadas pelo Projeto de Extensão "Saúde Mental na Atenção Básica: cuidado ao usuário em sofrimento psíquico e sua família”, desenvolvido pelo curso de Enfermagem da Universidade Federal de Santa Maria (UFSM), na área de abrangência de uma USF em um município no interior do Rio Grande do Sul, Brasil. O projeto está em andamento nesta unidade desde 2008. Atuam nesse projeto discentes e docentes do Curso de Graduação em Enfermagem da UFSM, residentes da Residência Multiprofissional Integrada do Sistema Único de Saúde da UFSM, outros profissionais que compõem a rede de saúde do município, bem como a equipe da USF.

A principal abordagem utilizada no projeto são as VDs, as quais ocorrem de acordo com a demanda existente e as prioridades discutidas com as equipes da USF, ouvindo-se principalmente as Agentes Comunitárias de Saúde (ACS). Tais visitas acontecem uma vez por semana. O grupo de acadêmicos se reúne com professor orientador, nas dependências da unidade, para a definição dos casos a serem visitados em cada dia e as condutas tomadas; posteriormente são realizadas as VDs, e então os visitadores retornam à USF, a fim de realizarem as anotações nos prontuários dos usuários. Há discussão dos casos com a equipe de profissionais da unidade, definição da conduta terapêutica, elaboração dos encaminhamentos necessários, mantendo a equipe da USF como corresponsável pelo acompanhamento dos casos. Este projeto de extensão possui registro no Sistema Integrado de Ensino (SIE) da Universidade Federal de Santa Maria com o nº 024038.

\section{(†)(-) Esta obra está licenciada sob uma Licença Creative Commons.}


Saúde mental na atenção básica: potencialidades para a construção de redes de atenção à saúde

\section{RESULTADOS E ANÁLISE}

Primeiramente, é importante destacar que o município em que o projeto é desenvolvido possui uma realidade singular no que tange à implantação da ESF. Iniciada em 2004, a ESF optou pela contratação terceirizada dos profissionais, decretada irregular em 2005 pelo Ministério Público do Trabalho (MPT), que determinou a demissão de todos os profissionais com esse tipo de vínculo empregatício, indicando concurso público. O concurso público das ACS aconteceu em 2008, mas os demais membros da equipe só foram concursados em 2011. Durante o período de 2008 a 2011 as equipes foram contratadas emergencialmente, ou seja, com contratos com prazo definido, de seis meses a um ano, impossibilitando a construção de vínculo com a população da área de abrangência. Esse fato é uma das justificativas de o projeto trabalhar mais próximo às ACS, pois são as que mais conhecem o território de abrangência da USF.

Optou-se por relatar a experiência agrupando alguns aprendizados, resultados e limites encontrados no desenvolvimento do projeto. Sem identificar os sujeitos envolvidos, pretendese discutir a saúde mental na $\mathrm{AB}$, destacando o potencial na construção da rede de atenção e no resgate da cidadania do usuário.

\section{Ressocializar: um processo que necessita vínculo e rede}

É histórico e contraditório o sentimento de medo que os profissionais manifestam pelo usuário com sofrimento psíquico. Exemplifica-se com o caso que foi conhecido como uma pessoa perigosa e ameaçadora, quando chegou à USF com um pé de cabra, sentou na cadeira em que se verifica pressão arterial (PA) e o colocou no colo. A equipe já conhecia o usuário e ficou afugentada, chamando a professora da enfermagem, responsável pelo projeto, para que verificasse a PA. Tratava-se de LS, masculino, 27 anos. Percebeu-se que o usuário estava mais para acuado do que para atacar, mas, apresentando-se desconexo e confuso, aquele pé de cabra tornava-se um risco. Naquele dia não foi possível realizar nenhuma abordagem sobre seu sofrimento psíquico. Mais tarde foi recebida na unidade a visita de duas pessoas da educação, professor e representante da coordenadoria regional, trazendo o problema da escola local. O jovem havia ameaçado o diretor e alunos e eles queriam interná-lo. Essa demanda gerou a possibilidade de a equipe acionar a Brigada Militar para buscá-lo. Foi sugerido, como

\section{Esta obra está licenciada sob uma Licença Creative Commons.}


Saúde mental na atenção básica: potencialidades para a construção de redes de atenção à saúde

uma alternativa, realizar uma VD. Os integrantes do projeto se dispuseram a realizá-la juntamente com a equipe, o que foi aceito.

No momento da VD percebeu-se que mãe também sentia medo do filho, pois aconselhou que se falasse baixo para ele não perceber a presença dos profissionais. A equipe estava ali para falar com LS, e não podiam esconder-se dele. O moço saiu do quarto e dirigiuse à equipe com palavras muito agressivas. Depois de alguns momentos de apreensão, foi possível desenvolver uma conversa e fazer alguns acordos. Descobriu-se que ele havia abandonado o tratamento farmacológico, pois não conseguira mais falar com a médica psiquiatra que o atendia no ambulatório municipal. Em sua fala ele revela ter vínculo somente com essa médica no serviço e só tomaria medicação novamente se fosse indicação dela. Foi proposto para ele retornar a usar a medicação, pois havia sido prescrita pela médica de sua confiança, e a equipe de saúde agendaria seu retorno. Ele cumpriu a sua parte e tomou o comprimido no momento da VD.

Depois da VD, ao retornar à USF, percebeu-se que a parte da equipe de saúde no acordo não seria muito fácil de realizar. $\mathrm{O}$ caso era conhecido no serviço de referência em questão como "o psicopata que abandonou o tratamento" e essa equipe havia decidido em reunião que não reagendaria consultas perdidas. Depois de muita negociação, foi conseguida uma vaga para alguns meses depois. O usuário voltou a usar a medicação regularmente e foi na consulta agendada com a psiquiatra.

O projeto mantém as VDs, acompanhando a família. LS estava terminando o Ensino Médio, mas havia abandonado os estudos. Em pouco tempo, voltou à escola e hoje consegue planejar o futuro, construindo a sua autonomia, sujeito da própria vida. Durante as visitas, eram identificadas as necessidades e ocupações em que ele tinha interesse, construindo assim a possibilidade de LS concluir os estudos, explorando a ideia de realizar o vestibular. Foi cultivado o gosto dele pelas plantas do jardim de sua residência e incentivado para que ele tivesse alguma atividade física. Destaca-se que o foco na saúde e não na doença permitiu um processo de reinserção social desse usuário, bem como minimizou o sofrimento dele e da família em relação à doença.

Com essa experiência, foi possível analisar algumas questões que interferem na construção da Rede de Atenção à Saúde (RAS) e no resgate de cidadania. Pode-se afirmar que, se tivessem seguido a sugestão de chamar a Brigada Militar, o vínculo com LS seria 
Saúde mental na atenção básica: potencialidades para a construção de redes de atenção à saúde

prejudicado, e a conduta, possivelmente, não seria a mesma. Enquanto o usuário em sofrimento psíquico representa uma ameaça aos profissionais e aos serviços de saúde, é abandonado por eles. Qual fato determina o outro? Será que, por ele ser agressivo, os profissionais se afastam? Ou será que ele torna-se agressivo porque os profissionais o abandonam? Com a continuidade do projeto percebeu-se que o abandono por parte dos profissionais aconteceu primeiro. O vínculo estabelecido com os participantes do projeto, somado ao suporte familiar e ao tratamento medicamentoso, revelou que é possível viver sem o risco de agressão. A escuta foi a ferramenta mais importante neste caso.

Outra análise que se precisa fazer é que o atendimento especializado, neste caso, com a psiquiatra, não é suficiente para a redução do sofrimento psíquico. Isso não é novidade na saúde mental. O modo médico-centrado, como este caso estava, sem o envolvimento e sensibilização de uma equipe interdisciplinar, não era eficiente para conduzir um projeto terapêutico que visasse à promoção da saúde e ao suporte ao usuário com o sofrimento psíquico e sua família. Também não é novidade para o SUS, pois sabemos que a integralidade só é possível em rede, um serviço sozinho não consegue dar conta disso (HARTZ e CONTANDRIOPOULOS, 2004).

Entretanto, a experiência com o projeto permite dizer que ainda é necessário apontar as lacunas no trabalho interdisciplinar e nas conexões dos serviços para que a integralidade e a RAS se concretizem. Presenciou-se a realidade do município em questão, no qual o atendimento em saúde mental do SUS está centrado em especialidade, não envolve a $A B$ e não estabelece conexões entre os pontos da rede de cuidado.

\section{Sequelas de uma história de abandono}

O projeto também encontrou o que foi chamado de sequela de uma história de abandono. Os integrantes do projeto foram convidados por uma ACS a visitar uma família em que, segundo ela, pai, mãe e filho eram deficientes mentais. A ACS revelou que havia recebido o caso com a sentença de que não havia nada a ser feito, ou seja, ela queria só dividir a angústia de assistir de mãos amarradas a uma situação chocante.

$\mathrm{Na}$ primeira VD realizada, mãe e filho esconderam-se ao ver os participantes do projeto, não foi possível estabelecer nenhum diálogo com eles, somente com o marido, que

\section{Esta obra está licenciada sob uma Licença Creative Commons.}


Saúde mental na atenção básica: potencialidades para a construção de redes de atenção à saúde

contou a história da família. Sua principal queixa foi de que os dois não dormiam e a esposa não se alimentava direito e não permitia que o filho se alimentasse. Nesse dia conferiu-se a terapia medicamentosa de todos e foi percebido que o pai, mesmo com uma doença neurológica que dificultava sua fala, conseguia ser autônomo e participar do cuidado de sua família. Durante o acompanhamento realizado por meio das VDs, percebeu-se que se tratava de uma senhora com história de sofrimento psíquico que se iniciou após o nascimento deste filho, então com 40 anos, e passou por inúmeras internações psiquiátricas, cronificando o adoecimento, o que os fez ficarem reclusos à casa, sem nenhum contato social, além do núcleo familiar.

A cronicidade do caso possivelmente deu-se pela falta de vínculo com serviço de saúde e também pela ausência de RAS. Foram 40 anos de abandono e muita vida a ser recuperada. $\mathrm{O}$ projeto encontrou muitos limites para atuar, pôde-se fazer pouco ainda com eles, mas muito mais do que nada. Em discussão com a equipe, em um primeiro momento, foi revista a terapia medicamentosa da mãe e do filho. Outro filho do casal, que residia na casa da frente, no mesmo terreno, cuidador principal de todos, se responsabilizou em iniciar o novo tratamento. Com as visitas posteriores, os integrantes do projeto viram resolutividade ao saberem que eles estavam se alimentando, dormindo melhor, e que a senhora iniciava um processo de ressocialização com a família, interagindo com os netos e filhos.

Apesar de o resultado ser tímido, pôde-se apreender com este caso que a Reforma Psiquiátrica demorou muito para ocorrer. Ainda existem sequelas de um tempo de tratamento hospitalocêntrico, desumano e fragmentado. Foi muito doloroso detectar o estado de abandono desta senhora, revelado pela sua higiene precária, magreza e confusão mental. Foi sabido que antes do parto ela era uma exímia faxineira e bordadeira. Hoje ela não gosta de falar sobre isso. Apesar dos poucos avanços, pode-se contar que hoje ela recebe os participantes do projeto e corresponde aos abraços. Também dizer que ações como as do projeto precisam existir e se multiplicar.

Diante das vivências proporcionadas pelo projeto, algumas reflexões são possíveis. Destaca-se como principal aprendizado e desafio a construção de redes de cuidado, que devem ser permeadas pelo compartilhamento de responsabilidades. Sabe-se que as ações em saúde mental na $\mathrm{AB}$ devem estar fundamentadas nos princípios do SUS e da Reforma Psiquiátrica, obedecendo ao modelo de redes de cuidado, bem como à atuação transversal

\section{Esta obra está licenciada sob uma Licença Creative Commons.}


Saúde mental na atenção básica: potencialidades para a construção de redes de atenção à saúde

com outras políticas específicas e que busquem o estabelecimento de vínculos e acolhimento. (BRASIL, 2011)

Nessa perspectiva, ressalta-se que o vínculo entre os participantes do projeto com a equipe da USF tornou possível uma responsabilização entre esses atores, uma vez que, de acordo com as necessidades dos sujeitos, são discutidos e planejados os planos de assistência. Defendeu-se que o cuidado em saúde mental na $\mathrm{AB}$ não fosse guiado por uma classificação nosológica, ou seja, o objetivo precisa estar voltado para aliviar sofrimento e não encaixar o usuário em um nome de patologia. Para isso, a adoção de uma clínica ampliada pode auxiliar, pois deixa tomar-se pelas diferenças do sujeito doente, reconhece os limites do universal para compreender o singular e produz um projeto terapêutico que leva em conta essas diferenças. Considerando a complexidade de vida do sujeito, o que inicialmente é um limite do saber pode ser transformado em possibilidade prática de intervenção (CUNHA, 2004).

Com o projeto avançou-se na possibilidade de diminuir o abandono dos casos acompanhados, evitando que o usuário ficasse sem uma equipe que se responsabilize por ele. Embasando-se na clínica ampliada em saúde mental, foi possível proporcionar a ampliação da inserção do usuário e sua família no planejamento da terapêutica. A construção da clínica ampliada em saúde mental acontece a partir da necessidade de transformação da atenção individual e coletiva para que outros aspectos do sujeito possam ser compreendidos e trabalhados, que não apenas o biológico (CUNHA, 2004).

Em consonância a isso, o Projeto Terapêutico Singular (PTS) busca a singularidade como elemento central de articulação da clínica com os sujeitos, é a partir dessa percepção da complexidade de cada caso que o profissional pode perceber que muitos determinantes do problema não estão ao alcance de intervenções pontuais e isoladas. Assim, percebe-se a necessidade da autonomia do sujeito no processo de reabilitação e promoção de sua saúde (BRASIL, 2007). Entende-se que o PTS deve ser construído em equipe, não pode ser proposto pelo projeto de extensão, pois corre-se o risco de não ter continuidade. Em vista disso, foram encontrados muitos limites na construção de PTS, devido às sucessivas trocas de equipe que ocorreram na USF, fruto da história de implantação da ESF no município.

Outro desafio é o entrelaçamento da rede de atenção, movimento que deve ser buscado para se garantir a integralidade da atenção. A estruturação da rede de atenção em saúde mental é fundamental para que aconteçam a Reforma Psiquiátrica e o alcance do seu eixo

\section{Esta obra está licenciada sob uma Licença Creative Commons.}


Saúde mental na atenção básica: potencialidades para a construção de redes de atenção à saúde

norteador, que é a inclusão dos usuários e o resgate da cidadania do indivíduo com transtorno psíquico (TENÓRIO, 2002). Com o projeto, em alguns momentos, foi possível a oportunidade de contar com o apoio matricial de equipes de saúde mental de um Centro de Atenção Psicossocial (CAPS). Tal dispositivo é importante para a construção de um arranjo organizacional no qual a equipe responsável, compartilha alguns casos com a equipe de saúde local. Esse compartilhamento produz corresponsabilização, que pode se efetivar por meio de discussões conjuntas de caso, intervenções também conjuntas com as famílias e comunidades ou em atendimentos compartilhados. A proposta reforça a intensão de excluir a lógica do encaminhamento e aumentar a capacidade resolutiva de problemas de saúde pela equipe local, possibilitando, gradativamente, a interdisciplinaridade e a ampliação da clínica na equipe (BRASIL, 2003).

\section{CONSIDERAÇÕES FINAIS}

A experiência com o projeto de extensão, desenvolvendo ações de saúde mental na $\mathrm{AB}$, é de fundamental importância para a academia, para o serviço e, principalmente, para o usuário e sua família. $\mathrm{O}$ processo de formação em saúde que possibilita a oportunidade de vivenciar essas práticas ganha em aprofundamento na complexidade da atenção integral em saúde mental, se comparado a cursos que só oferecem a vivência dentro de hospitais e/ou CAPS. O serviço, no caso do município em questão, também é beneficiado, pois aproxima as equipes dos princípios da Reforma Psiquiátrica e permite a proximidade com usuários que antes estavam somente vinculados a outros serviços.

Destaca-se que o projeto faz diferença na vida dos usuários e suas famílias, pois possuir um trabalhador de saúde, no caso um profissional em formação, como referência, facilita o diálogo com a ESF e com os demais pontos da rede. Além disso, é possível afirmar que interfere-se positivamente na promoção da autonomia dos usuários e os incluindo, com seus contextos e singularidades, na elaboração de sua proposta terapêutica.

As ações em saúde mental no cuidado contextualizado são parte essencial para a efetivação da rede de atenção à saúde mental. A VD e o acompanhamento singular e subjetivo apresentam-se como ferramentas resolutivas dos casos encontrados no território de abrangência do projeto.

\section{Esta obra está licenciada sob uma Licença Creative Commons.}


Saúde mental na atenção básica: potencialidades para a construção de redes de atenção à saúde

Enfrentaram-se alguns limites no desenvolvimento do projeto, que foram a constante troca de equipes da USF, que no momento já estão concursadas, e também as lacunas da rede de atenção à saúde no município. As articulações possibilitadas com o projeto reforçam que a construção das redes de cuidado necessita dos espaços micropolíticos para acontecer.

\section{REFERÊNCIAS}

BRASIL. Ministério da Saúde. Secretaria de Atenção à Saúde/DAPE. Saúde Mental no SUS: acesso ao tratamento e mudança do modelo da atenção. Relatório de Gestão 20032006. Brasília: Ministério da Saúde; 2007.

BRASIL. Ministério da Saúde. Secretaria de Atenção à Saúde. DAPE. Coordenação Geral de Saúde Mental. Reforma psiquiátrica e política de saúde mental no Brasil. Documento apresentado à Conferência Regional de Reforma dos Serviços de Saúde Mental : 15 anos depois de Caracas. OPAS. Brasília, novembro de 2005.

BRASIL. Lei $N^{\circ} 10.216$ de 06 de abril de 2001. Dispõe sobre a proteção e os direitos das pessoas portadoras de transtornos mentais e redireciona o modelo assistencial em saúde mental. Antigo Projeto de Lei Paulo Delgado.

BRASIL. Departamento de ações programáticas estratégicas. Departamento de atenção básica coordenação geral de saúde mental. Coordenação de gestão da atenção básica. Saúde Mental e Atenção Básica o Vínculo e o Diálogo Necessários. Coordenação de Saúde Mental e Coordenação de Gestão da Atenção básica, n ${ }^{\circ}$ 01/03

BRASIL, Ministério da Saúde. Secretária de Atenção à Saúde. Núcleo Técnico da Política Nacional de Humanização. Clínica ampliada, equipe de referência e projeto terapêutico singular/ Ministério da Saúde, Secretária de Atenção à Saúde, Núcleo Técnico da Política Nacional de Humanização- 2.ed. - Brasília: Ministério da Saúde, 2007.

BRASIL, Ministério da Saúde. Secretaria de Atenção à Saúde. Departamento de Atenção Básica. Política Nacional de Atenção Básica / Ministério da Saúde, Secretaria de Atenção à Saúde, Departamento de Atenção à Saúde. - Brasília: Ministério da Saúde, 2011.

CAMPOS, Gastão Wagner de Sousa et al. Tratado de saúde coletiva. São Paulo: Hucitec; 2006.

\section{Esta obra está licenciada sob uma Licença Creative Commons.}


Saúde mental na atenção básica: potencialidades para a construção de redes de atenção à saúde

CUNHA, Gustavo Tenório. A Construção da Clínica Ampliada na atenção Básica. Campinas, SP, 2004.

GOFFMAN, Erving. Manicômios, Prisões e Conventos. São Paulo: Perspectiva, 2003.

LABATE, Renata Curi ; GALERA, Sueli Aparecida Frari ; AVANCI, Rita de Cássia. Visita domiciliária: um olhar da enfermagem psiquiátrica. Revista Brasileira de Enfermagem, v.57, n.5, p:627-628, 2004

REINALDO, Amanda Márcia dos Santos, ROCHA, Ruth Milyus. Visita domiciliar de Enfermagem em Saúde Mental: idéias para hoje e amanhã. Revista Eletrônica de Enfermagem, v.4, n.2, p:36-41, 2002.

TENÓRIO, Fernando. A reforma psiquiátrica brasileira, da década de 1980 aos dias atuais; história e conceitos. Revista História, Ciências, Saúde, v.9, p:25-9, 2002

\section{AGRADECIMENTOS}

Agradecemos à Universidade Federal de Santa Maria e ao Fundo de Incentivo à Extensão, cujos apoios foram imprescindíveis para a realização do projeto de extensão aqui apresentado. 